die die Eiweißkörper durch die Bestrahlung erfahren ${ }^{5}$ ). Doch kann das hier nicht näher erörtert werden*). -

Fassen wir zum Schluß noch einmal zusammen! Nach unserer Auffassung lrommt die Aufhebung der normalen Stabilität der Blutkörperchensuspension in der Schwangerschaft und bei Krankheiten dadurch zustande, daß in der Adsorptionshülle der Blutkörperchen Globuline mehr oder weniger an die Stelle von Albumin treten, und da der isoelektrische Punkt der Globuline, also die Reaktion, bej der ihre Flockungstendenz maximal ist, der neutralen Reaktion des Blutes näher liegt, als der isoelektrische Punkt des Albumins, so wächst auch bei den Blutkörperchen, die mit mehr Globulin behaftet werden, die Tendenz zur Flockung oder Agglutination. Die Wirkung der Eiweißkörper richtet sich aber, abgesehen von der Lage ihres isoelektrischen Punkts, auch noch nach ihrem Lösungszustand, insbesondere nach ihrer Viscosität, durch die der Blutkörperchenoberfläche ein verschiedenes $\mathrm{Ma} \beta$ von Klebrigkeit erteilt wird.

Lit e r a tu r: ${ }^{1}$ ) FÄHRaEus, Biochem. Zeitschr. 89, 355. 19I8. 2) FÂ hraeus, Acta med. Scand. Vol. 55. I921. - 3) LinzenMeter, Pflügers Arch. f. d. ges. Physiol. I8I, I69. 1920 und I86, 272. I92I, - ${ }^{4}$ ) ReITSTÖTTER, Zeitschr. f. Immunitätsforsch. u. exp. Therap. 30, 507. I920. - 5) R. Mond, Pflügers Arch. f. d. ges. Physiol, 196, 540. 1922.

\section{ÜBER DIE BETEILIGUNG DER ANTAGONISTEN AN DEN WILLKÜRBEWEGUNGEN DES MENSCHEN**).}

\section{(Vorläufige Mitteilung.)}

Von

Dr. KuRT WACHHOLder.

Aus dem Physiolog. Institut der Universität Breslau.

Es ist eine alte, vielumstrittene Frage, ob an der Ausführung unserer Bewegungen neben den Muskeln, weiche das Glied direkt in die beabsichtigte Lage hinziehen, den sog. Agonisten, noch diejenigen Muskeln, welche die entgegengesetzte Zugrichtung haben, die sog. Antagonisten aktiv beteiligt sind oder nicht. Dieses Problem hat bei seiner gleich großen theoretischen und praktischen Bedeutung von den verschiedensten Seiten zahlreiche Bearbeitungen gefunden, ohne jedoch bisher eine allgemein anerkannte Lösung gefunden zu haben. Der alten Duchenneschen Lehre'), $\mathrm{da} B$ die Antagonisten durch ihre Kontraktion die Bewegungen mäBigen und regulieren, steht die andere besonders von H. E. HerING ${ }^{2}$ ) vertretene Anschauung gegenüber, daß die Antagonisten sich ${ }_{r}$ passiv verhalten, daß ,,wahre Antagonisten

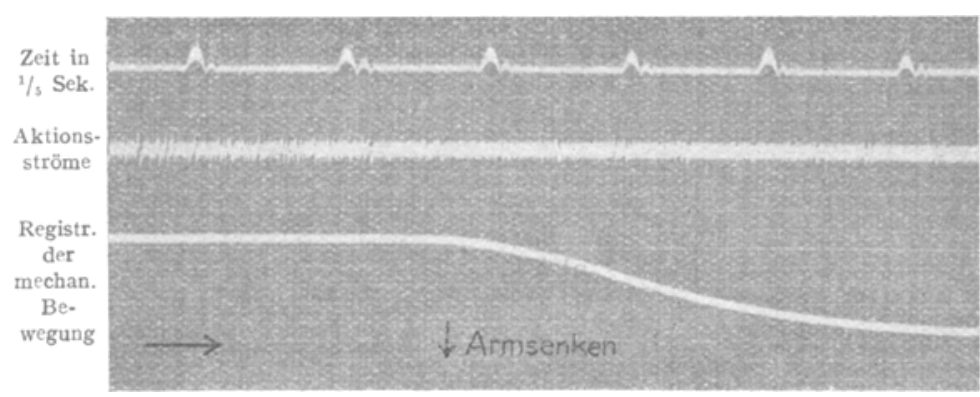

Abb. I. Aktionsströme des M. Deltoideus bei gehobenem Arm und beim Sinkenlassen des Armes.

nicht gleichzeitig innerviert werden." Unter dem Eindrucke der Sherringtonschen Forschungen über die reziproke Innervation dachte man vielfach an eine vollkommene Hemmung der Antagonisten. Die neuesten Untersuchungen von BeTHE ${ }^{3}$ ) an Sauerbruchoperierten zeigen, daß tatsächlich auch beim

*) Siehe überhaupt die demnächst erscheinenden ausführlichen Veröffentlichungen von MOND, KANAI und LEY in Pflügers Archiv f. d. ges. Physiol.

**) Nach einem Vortrage, gehal ten bei dex Tagung der Gesellsch. deutsch. Naturf. u. Arzte in Leipzig am 22. September r922.
Menschen eine reziproke Innervation vorhanden ist. Die Arbeiten von Gregor und SCHILDER ${ }^{\circledR}$ ) sowie von F. H. LEWY ${ }^{5}$ ) sprechen jedoch zum Teil gegen eine Untätigkeit bzw. vollkommene Hemmung der Antagonisten.

Vorliegende Untersuchurgen benutzen den schon von den letztgenannten Autoren beschrittenen Weg, aus dem Vor-

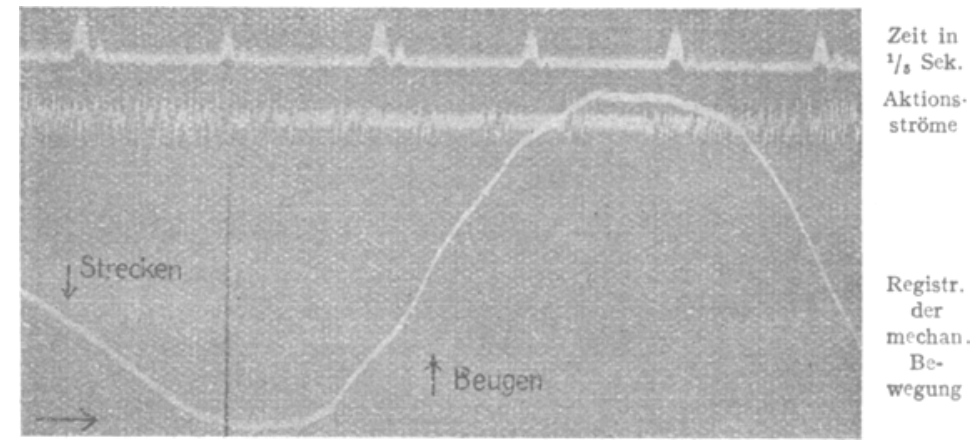

Abb. 2. "Aktionsströme des M. Extensor carpi radialis bei rhythmischem Strecken und Beugen der Hand.

handensen bzw. Fehlen von Aktionsströmen im Antagonisten zu schließen, ob dieser an der Ausführung einer Bewegung aktiv beteiligt ist oder nicht. Zur Ableitung der fraglichen Ströme wurden in die Muskeln eingestochene Nadelelektroden aus Neusilber benutzt. Kontrollversuche ergaben die Möglichkeit einer praktisch isolierten Ableitung vom Antagonisten. Die $2 \mu$ dicke Pt-Saite des Galvanometers wurde zur getreuen Wiedergabe der Frequenz der Aktionsströme sehr stark, bis an die Grenze der Aperiodizität gespannt.

Zuerst wurde die einfachere Frage geprüft: Welche Aktionsströme treten bei allmählicher Kontraktion und nachfolgender allmählicher Erschlaffung in einem Muskel auf, wenn seine Innervation möglichst unbeeinflußt ist von der anderer Muskein, besonders von der seines Antagonisten? Als Beispiel seien die Aktionsströme des M. deltoideus besprochen bei relativ langsamem Heben und Senken des Armes. Wenn der Arm gehoben wird und solange er gehoben bleibt, treten im Deltoideus Aktionsströme auf, die mit einer Frequenz von I5O-I70 Sek. ununterbrochen aufeinanderfolgen. Wenn der Arm nunmehr langsam gesenkt wird (Abb. I Senkung der unteren Linie), so werden die Aktionsströme kleiner bis zum völligen Verschwinden, um nach einer kurzen Pause der Stromlosigkeit von neuem aufzutreten, wieder zu verschwinden, wieder aufzutreten usw. Diese periodenweise auftretenden Aktionsströme werden immer schwächer, bis sie gegen Ende der Erschlaffung eben nur noch sichtbar sind. Die einzelnen Perioden der Aktionsströme folgen aufeinander in oft recht regelmäßigen Abständen von durchschnittlich 1/10 Sek., sie bestehen jedesmal aus einer Gruppe von mehreren Einzelschwankungen, unter denen meistens eine oder mehrere größere Schwankungen hervortreten. Die Frequenz dieser Einzelschwankungen ist annähernd dieselbe wie diejenige während des Armhebens, also r5o-r7o Sek. In den Pausen zwischen diesen Perioden stärkerer Saitenschwankungen ist in vielen Fällen der Saitenschatten ganz glatt, manchmal sind jedoch auch hier kleine Zacken zu sehen. Dieses periodenweise Verschwinden und Wiederauftreten der Aktionsströme, das zuerst von P. HoFrmanN ${ }^{6}$ ) beobachtet wurde, ist ganz charakteristisch für die Phase der Erschlaffung, es wurde in allen daraufhin untersuchten Muskeln bei einer Anzahl von Bewegungen gefunden.

Wir betrachten nunmehr den bei den allermeisten unserer Bewegungen vorliegenden Fall, daßB ein Paar von antagonistisch zueinander wirkenden Muskeln in Wechselwirkung steht und speziell, ob sich diese Wechselwirkung durch irgendeine Veränderung in den Aktionsströmen zumal während der Erschlaffungsperiode des untersuchten Muskels kundgibt. Abb. 2 zeigt eine Aufnahme der Aktionsströme des M. Extensor carpi radialis bei rhythmischer Beugung und Streckung der Hand, während die mäßig gebeugten Finger 
sich nicht bewegten. Der Unterarm war fest unterstützt und nur die Hand beweglich. Wenn die Hand gestreckt wird, der untersuchte Muskel also Agonist ist - Sinken der unteren Linie in Abb. 2 - treten in ihm ununterbrochen aufeinanderfolgend dieseiben frequenten Aktionsströme auf wie im Deltoideus. Wird die Hand nunmehr gebeugt - Steigen der unteren Linie in $\mathrm{Abb} .2$-, so verschwinden die Aktionsströme im Extensor carpi radialis, der jetzt also Antagonist ist, nicht, sondern treten mit derselben Periodenbildung auf, wie sie im langsam ohne reziproke Innervation erschlaffencen Deltoideus oben beschrieben wurde. An der Bewegungskurve sieht man deutlich, wie zeitlich kurz nach jeder einzelnen Aktionsstromgruppe die Kurve weniger steil ansteigt, die Beugung also durch eine kurze Kontraktion des Antagonisten gebremst und verzögert wird, um dann wieder steiler $\mathrm{zu}$ verlaufen bis zur nächsten. Aktionsstromgruppe. Dasselbe periodenweise Auftreten von Aktionsströmen konnte in den anderen untersuchten Muskeln, so im Biceps und Triceps bei Beugen und Strecken des Unterarmes festgestellt werden, wenn diese als Antagonisten dienten. Es sind also vom Antagonisten Aktionsströme ableitbar, die in ihrem" Aussehen, speziell in der Bildung von Perioden weitgehend denen entsprechen, welche im Agonisten während des Stadiums allmählicher Erschlaffung auftreten. Das im Falle einer Untätigkeit oder völligen Hemmung des Antagonisten zu erwartende gänzliche Fehlen von Aktionsströmen in ihm wurde nicht beobachtet. Der Befund beweist, daß nicht nur den Agonisten, sondern auch den Antagonisten bei der Ausführung unserer Bewegungen eine aktive Rolle im Sinne der Duchenneschen Lehre zuzusprechen ist, und zwar erfolgt die Innervation bzw. Kontraktion des Antagonisten nicht kontinuierlich, sondern stoßweise in. Abständen von etwa $1 / 10$ Sek., einerlei ob die Bewegung schnell oder langsam ausgeführt wird.

Die nicht ganz glatte, sondern durch Verzögerungen unterbrochene Ausführung der Handbewegungen wurde schon von $\mathrm{PFAHL}^{1}$ ) graphisch registriert, der sie auch schon richtig erklärte durch die Annahme einer stoßweisen Kontraktion des Antagonisten. Der Nachweis des periodenweisen Auftretens von Aktionsströmen im Antagonisten und dessen zeitliche Úbereinstimmung mit den Verzögerungen der Bewegung bestätigt diese Vermutung von PFAHL.

Wenn diese Ergebnisse auch die Anschauung vom passiven Verhalten der Antagonisten nicht bestätigen, so beweisen sie dagegen meines Erachtens durchaus nicht, daß die Lehre von der reziproken Innervation keine Anwendung auf die menschlichen Bewegungen finden darf. Der Begriff der reziproken Innervation setzt nicht völlige Hemmung des Antagonisten voraus, vielmehr ist die Hemmung nach den Untersuchungen von SHERRINGTON ${ }^{8}$ ) weitgehend abstufbar und nach der Halbzentrentheorie von GRAHAM BROWN sind anch gleichzeitige Kontraktionen im Agonisten und Antagonisten erklärbar $\left.{ }^{9}\right)$. Die theoretische Deutung des experimentellen Befundes und der Versuch einer Analyse der beobachteten Periodenbildung der Aktionsströme bleibt jedoch weiteren Untersuchungen und einer späteren ausführlicheren Darstellung vorbehalten, desgleichen eine Erörterung der Schlußfolgerungen, welche sich für die pathologischen Störungen der Bewegungskoordination ergeben.

Literatur: ${ }^{1}$ ) Duchenne, Psychiol. der Bewegungen übers. v. Wernicke. - 2) H. E. Hering, Pflügers Arch. f. d. ges. Physiol 70, 559. I898. - 4) BETHE, Pflügers Arch. f. d. ges. Physiol. x94, 77. 1922. - ${ }^{4}$ ) GREGOR u. ScHÜLDER, Zeitschr. f. d. ges. Neurol. u. Psychiatr. 14, 359. I913. - 5) F. H. LEwy, Zeitschr. f. d. ges. Neurol. u. Psychiatr. 58, 310. I920. $\left.-{ }^{6}\right)$ P. Hofrmann, Zeitschr. f. Biol. 73, 247. 1921. - 7) PFaHL, Pfügers Arch. f. d. ges. Physiol. I88, 298. I92 I. - 8) Sherrington, Quart. Journ. of exp. Physiol. I, 67. 1908. - 9) WACHHoHLer, Zeitschr. f. allg. Physiol. 20, I6I. I922.

\section{DIREKTE MUSKELPRÚFUNG ZUR PROGNOSE DER POLIOMYELITISCHEN LÄHMUNGEN BALD NACH DEM AKUTEN ANFALL*.)}

\author{
Von
}

Dr. Philipp ErLacher, Graz.

Kinderklinik Graz: Vorst. Prof. HAMBURGER; Chirurg.-orthopadische Abteilung: (Leiter Doz. ERLACHER.)

Wenn nach Ablauf des akut fieberhaften Anfalles und Rückgang der bedrohlichen Erscheinungen der Kinderlähmung eine oder mehrere Extremitäten vollkommen gelähmt erscheinen, richten die Eltern die bange Frage an uns, wieviel von dieser Lähmung zurückbleiben, wieviel sich wieder zurückbilden wird. Wir konnten bisher nur ganz allgemein antworten, daB in den ersten Wochen und Monaten wesentliche Rückgänge der Lähmungen beobachtet werden und nur die Lähmungen, die nach einem Jahre noch immer keine Besserung zeigen, voraussichtlich als bleibend anzusehen sind. Die faradische Untersuchung in der bisherigen Art versagt in dieser Beziehung vollkommen, die Muskeln sprechen weder direkt noch indirekt faradisch an, galvanisch kann E.-A.-Reaktion festgestellt werden und die galvanische Erregbarkeit sinkt allmählich. SpItzy hat darauf hingewiesen, daß das rasche Ansteigen der Milliamperezahl, die zur Erregung des Muskels notwendig ist, uns einen Fingerzeig in der Beurteilung der Lähmung gibt. Wenn die normale Milliamperezahl im gelähmten Muskel nach zwei Monaten bereits das Doppelte, nach 4-5 Monaten das Dreifache und darüber beträgt, so besteht nach seiner Erfahrung keine Hoffnung auf Spontanregeneration. Aber auch die Aussichten einer Nervenplastik werden immer geringer, je weiter die Degeneration im Muskel fortschreitet; ,wenn gar keine direkte Erregbarkeit da ist, ist sie aussichtslos." Diese Ansicht war auf die bis dahin allgemein verbreiteten und anerkannten neurologischen Lehrsätze über die faradische und galvanische Erregbarkeit von Nerven und Muskeln bei Lähmungen aufgebaut. Nun haben aber die Beobachtungen von PeRThes ${ }^{1}$ ) am freigelegten Muskel gelegentlich von Nervenoperationen darin gründlich Wandel geschaffen. Er konnte am Menschen nachweisen, daß , die gelähmten Muskeln in der Zeit von zwei Monaten bis $I^{1} / 2$ Jahren nach Abschluß des zugehörigen Nerven deutliche faradische Erregbarkeit zeigten“. "Niemals wurde der freigelegte Muskel innerhalb des ersten Jahres nach Durchtrennung des zugehörigen Nerven für den faradischen Strom völlig unerregbar gefunden, trotzdem die Muskeln sich bei der Untersuchung durch die Haut regelmäßig bei faradischer Reizung als absolut reaktionslos erwiesen hatten." Die Zuckung war acht. Wochen nach der Durchtrennung noch „,blitzartig", im siebenten Monat war sie träge, ,wurmartig", nach achtzehn Monaten zwar schwach, aber deutlich. Die histologische Untersuchung eines seit $6^{1} / 2$ Monaten gelähmten Muskels ergaben ,zahlreiche sehr schmale Fasern, welche in dieser Art beim erwachsenen Menschen nicht vorkommen dürften (vgl. meine diesbezügl. Befunde weiter unten. E.). Daneben fanden sich zahlreiche Fasern, deren Inhalt degeneriert ist und keine Querstreifung aufweist".

Angeregt durch diese Mitteilung von Perthes habe ich dann einen früheren Gedanken wieder aufgenommen, mittels direkt durch die Haut in den Muskel eingestochene Nadeln die direkte Erregbarkeit des Muskels festzustellen und diese Erregbarkeit vor allem zu diagnostischen $Z$ wecken auszubauen ${ }^{2}$ ). $\mathrm{Da} \beta$ es auf diese Weise möglich ist, eine wesentlich genauere Übersicht über die Ausdehnung einer Lähmung verschiedenster Atiologie zu bekommen, habe ich schon in meiner ersten Mitteilung (Igrg) hervorgehoben. Inzwischen habe ich die Methode vielfach angewendet und hatte auch Gelegenheit, bei mehreren Fällen von angeborener Plexuslähmung oder Schulterlähmung, wie sie vielleicht richtiger heißen sollte, meine direkte Nadelprüfung durchzuführen. Dabei konnte ich feststellen, daß sich diese Methode auch am Säugling sehr gut anwenden läßt, und gleichzeitig die neurologische Ansicht, $\mathrm{da} \beta$ der Neugeborene bei elektrischer Untersuchung ànders

*) Auszugsweise vorgetragen am Orthopäden-KongreB Breslau Ig22. 